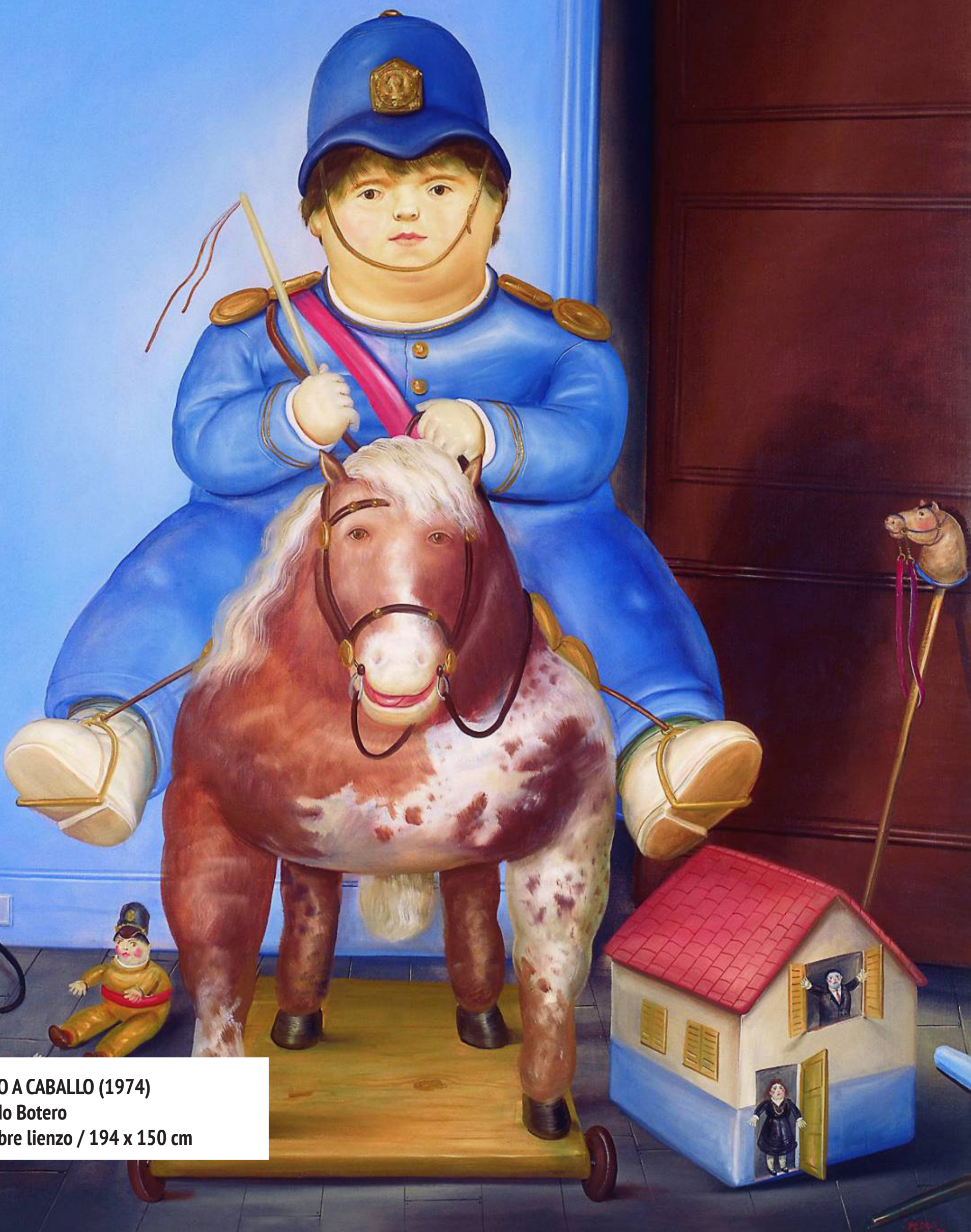




\section{PROYECTOS INTEGRADORES: VINCULACIÓN DE LA EDUCACIÓN SUPERIOR TECNOLÓGICA CON LAS MICROEMPRESAS}

\section{INTEGRATIVE PROJECTS: LINKING HIGHER TECHNOLOGICAL EDUCATION WITH MICROENTERPRISES}

\section{RESUMEN}

Los programas de ingeniería del Tecnológico Nacional de México tienen su diseño curricular sustentado en competencias basadas en una educación integral, considerando a la investigación como una estrategia útil para vincular a los institutos tecnológicos con el entorno local, regional y nacional. Los Proyectos Integradores (PI) forman parte de las estrategias para generar esa vinculación desde dos ámbitos: el desarrollo de competencias por parte de los estudiantes y la contribución al desarrollo social. En este ámbito es necesario identificar el impacto que genera en las microempresas la aplicación de los Pl; para ello, se realiza en la ciudad de Poza Rica del Estado de Veracruz, México, su aplicación a microempresas, utilizando la metodología sugerida por el mismo Tecnológico Nacional de México; la aplicación de técnicas de ingeniería que son consideradas en el diseño curricular de cada asignatura para el cumplimiento de las competencias, y determinar el nivel de participación de los empresarios mediante la aplicación de encuestas. Lo anterior nos lleva a conocer el impacto que produce la vinculación de la educación superior tecnológica con las microempresas locales y, por otra parte, se identifica si existe beneficio para ellas en lo referente a la planificación y organización de sus actividades para contribuir en su desarrollo.

PALABRAS CLAVE: Educación - Proyectos - Tecnología - Integración - Desarrollo - Microempresa.

\section{MARIBEL MARTÍNEZ LEE \\ CELSO RAMÓN SARMIENTO REYES}


os planes y programas de estudio del Tecnológico Nacional de Mé-xico se sustentan en un diseño curricular del Modelo Educativo para el Siglo XXI: La Formación y Desarrollo de Competencias Profesionales, basados en la educación integral y el desarrollo de las competencias en la formación de profesionistas. Se afirma que ésta es una forma de generar conocimientos pertinentes y de actualidad, que sirve para enriquecer el acervo cultural y considera a la investigación como una estrategia útil para vincular a los Institutos Tecnológicos con el entorno regional, nacional y mundial con la intención de contribuir al desarrollo social que involucra a todos los sectores de la sociedad. Específicamente en el caso del sector empresarial, el Tecnológico Nacional de México (TecNM) sugiere la aplicación de los Proyectos Integradores (PI) para involucrar a las empresas y estudiantes, incidiendo en la formación y desarrollo de competencias profesionales de estos últimos.

El contacto con las empresas, en específico las microempresas, permiten a los estudiantes aplicar los conocimientos adquiridos en su formación académica y fortalecer sus competencias de formación profesional. En este sentido, las prácticas profesionales se han convertido en una actividad académica con la que los estudiantes se vinculan con organizaciones a partir de asignaturas, asesorados por un profesor-tutor que les facilita la práctica en el mundo laboral.

\section{PLANTEAMIENTO DEL PROBLEMA}

La educación superior tiene un reto muy importante con la sociedad al ser considerada como motor de cambio de una nación; aunado a esto, el mundo

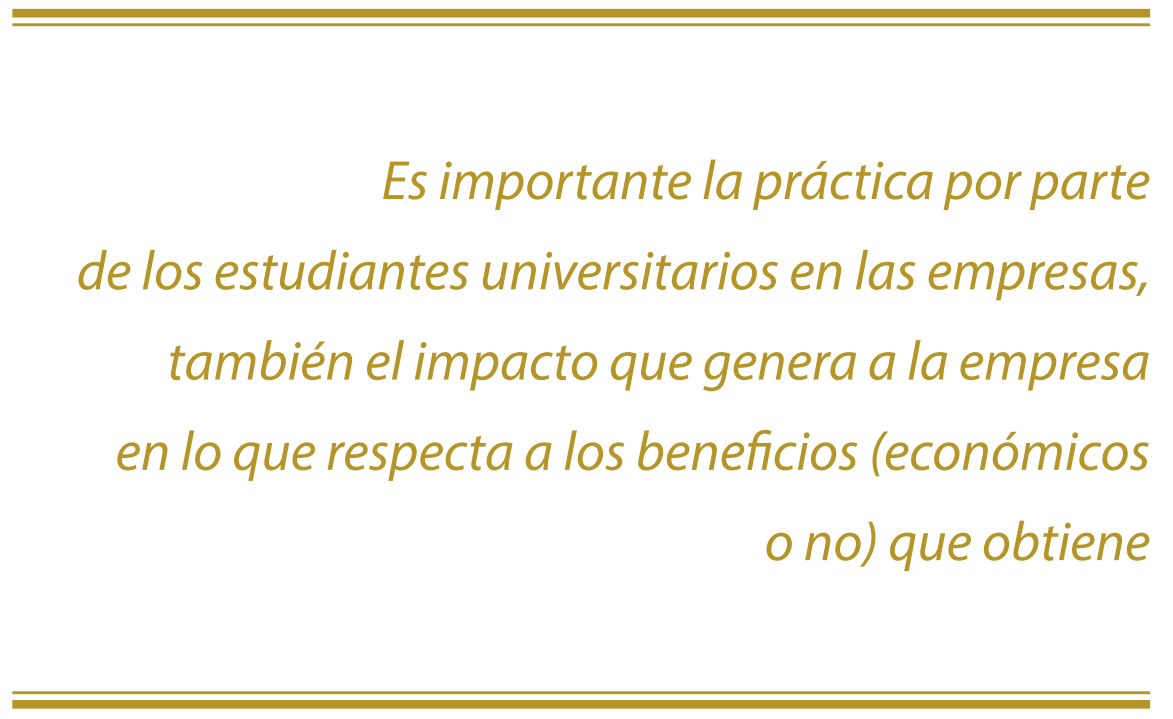

globalizado al que se enfrentan exige de los estudiantes una mejor preparación para el mundo laboral.

En el caso de la educación superior tecnológica, la exigencia que ésta enfrenta, es la demanda en diversos sectores de egresar ingenieros calificados para la toma de decisiones y la solución de problemas de manera eficaz y eficiente.

Al respecto, el Modelo Educativo para el siglo XXI: Formación y Desarrollo de Competencias Profesionales orienta el proceso educativo superior tecnológico central en la formación de profesionales que impulsen la actividad productiva en cada región del país, la investigación científica, la innovación tecnológica, la transferencia de tecnologías, la creatividad y el emprendimiento para alcanzar un mayor desarrollo social, económico, cultural y humano (TecNM 20014).

En este contexto, los PI son una herramienta importante para lograr que los estudiantes obtengan sus competencias a través de la práctica en el sector empresarial, coadyuvando con esto al desarrollo social.
Si bien es importante la práctica por parte de los estudiantes universitarios en las empresas, también lo es el impacto que les genera a las empresas la participación de los estudiantes en el desarrollo de ellas; es decir, que no únicamente debe considerarse como participación social o como contribución a la formación integral y profesional del estudiante, sino también el impacto que genera a la empresa la participación de los alumnos en lo que respecta a los beneficios (económicos 0 no) que obtiene. Lo anterior surge del siguiente cuestionamiento: ¿Qué impacto genera la aplicación de los PI en las microempresas?

\section{OBJETIVO GENERAL DE LA INVESTIGACIÓN}

Identificar el impacto que genera la aplicación de los PI en microempresas de Poza Rica, Veracruz, y en la vinculación entre sectores.

\section{OBJETIVOS ESPECÍFICOS DE LA INVESTIGACIÓN}

- Aplicar los PI a las microempresas de Poza Rica.

- Determinar el impacto de los PI.

- Aplicar encuestas a los empresarios designados. 


\section{HIPÓTESIS}

La aplicación de los PI en las empresas genera beneficios para ellas en la planificación y organización de sus actividades, lo cual se ve reflejado en la optimización en el uso de los recursos.

\section{JUSTIFICACIÓN}

\section{DE LA INVESTIGACIÓN}

En un escenario de constante lucha de las empresas por permanecer en el mercado, se vuelve fundamental la mejora continua en cada una de sus áreas. Sin embargo, en ocasiones, la falta de conocimiento 0 de recursos económicos nos les permite realizar trabajos para identificar áreas mejorables que podrían resolver problemas, aumentar ganancias y, por ende, mantenerse en el mercado. Por otro lado, los estudiantes de educación superior tecnológica requieren desarrollar sus competencias mediante la aplicación de técnicas de ingeniería en escenarios reales, ya que la literatura disponible no es suficiente para obtener toda la información que se requiere para aplicar dichas herramientas y detectar áreas de oportunidad y mucho menos poder presentar estrategias en base a datos reales. Asimismo, la falta de vinculación de los estudiantes con las empresas nos les permite el desarrollo de las competencias generales y específicas.

Teniendo como referencia lo anterior, surge la inquietud de contribuir al desarrollo, mejoramiento o estabilidad en el mercado de las microempresas mediante la educación tecnológica superior, misma que es considerada como uno de los principales elementos que influyen de forma directa en el desarrollo de una nación, por lo que se considera que una forma de contribuir a ello es mediante la vinculación

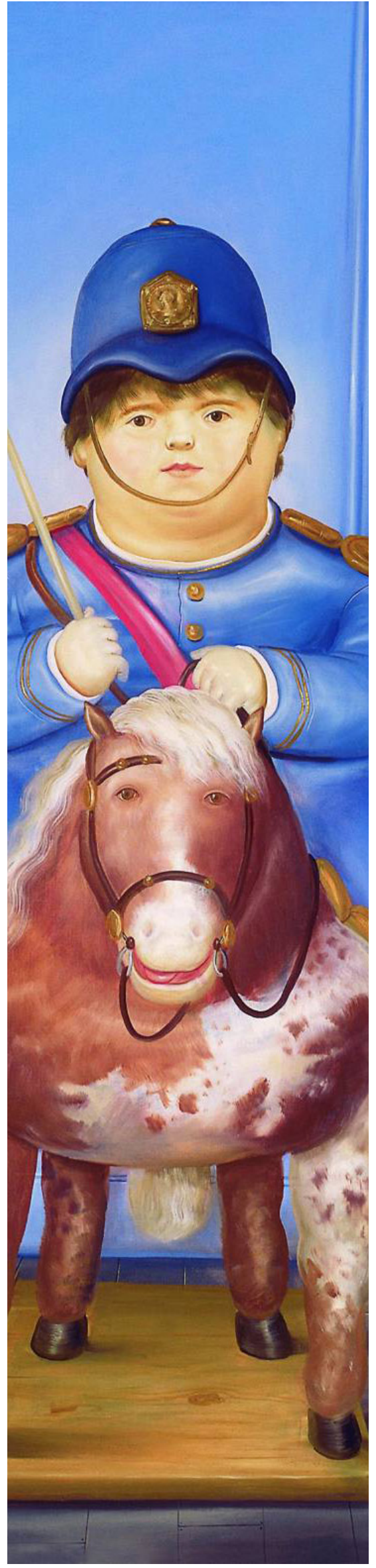

de la educación superior tecnológica con las microempresas, en donde los alumnos puedan detectar áreas de mejora y presentar estrategias para que el empresario las analice y pueda aplicar en relación con su disponibilidad financiera y material, así como evaluar el impacto de las mismas en su negocio. Con esto, la educación superior tecnológica se incorpora activamente a las iniciativas de desarrollo local, a través del fortalecimiento de las microempresas.

La educación superior ha tenido que cambiar un sistema de enseñanza y aprendizaje por objetivos y transformarlo a un sistema por desarrollo de competencias, este último consiste principalmente en los factores saber, saber hacer, saber ser, lo cual conlleva a que no únicamente en la educación profesional se obtiene conocimiento, sino que también deben aplicarse los conocimientos a su entorno (TecNM 2014). Una de las formas para realizarlo es mediante los proyectos integradores (PI), en los cuales se mejora la preparación de los estudiantes y en esa preparación, ocupa un lugar especial el Practicum, ya que desempeña un papel clave en el desarrollo de las competencias que permitan la transferencia y movilización de conocimientos a situaciones de trabajo (Molina Ruíz, 2007).

Un proyecto integrador es una estrategia didáctica que consiste en realizar un conjunto de actividades articuladas con un inicio, un desarrollo y un final, con el propósito de identificar, interpretar, argumentar y resolver un problema del contexto, y con esta dinámica contribuir a formar una 0 varias competencias del perfil de egreso, teniendo en cuenta el abordaje de un 
problema significativo del contexto disciplinar, investigativo, social, laboral, profesional, etc. (López Rodríguez, 2012).

Los proyectos integradores se incorporan a la Educación Superior Tecnológica como una estrategia curricular que permite generar una nueva vía para que los estudiantes desarrollen competencias (específicas y genéricas) a lo largo de su trayectoria en el instituto, lo que significa que debe de contemplar oportunidades para aprender a actuar de forma integral y no individualizada. Todo proyecto busca abordar problemas en el contexto, y en ese sentido es la estrategia más integral para la formación y evaluación de las competencias (Tobón S. y., 2010b).

Un proyecto integrador moviliza los conocimientos que permitan la vinculación de instituciones educativas y la sociedad en su conjunto, donde los saberes del estudiante trascienden el ámbito escolar y le permitan acumular experiencia a través de la respuesta a prácticas predominantes y emergentes de su contexto, al mismo tiempo que favorece el desarrollo de la sociedad misma, acorde a la misión y visión del Tecnológico Nacional de México (TecNM, 2014). Es necesario abordar para este trabajo el tema de microempresas que son consideradas de gran importancia para la economía nacional por las aportaciones a la producción y distribución en los bienes y servicios, así como también por los empleos que generan y la flexibilidad de adaptarse a los cambios tecnológicos, buscando e innovando a través de los años.

En la actualidad las microempresas se consideran como las empresas que na- cen, se desarrollan y, después de algún tiempo, se enferman y dejan de operar. Ante un panorama competitivo, la presión que experimentan las organizaciones para mantenerse en el mercado y cumplir con las expectativas de los clientes tanto internos como externos, es muy grande (Saldaña, 2005). Y de esto se desprende la colaboración del sector educativo superior tecnológico, posibilitando que los estudiantes contribuyan al desarrollo de estas.

Para el caso del desarrollo local, Alburquerque (2002) lo considera como el proceso en el cual los actores 0 instituciones locales se movilizan para apoyar las diferentes acciones, tratando de crear, reforzar y preservar actividades y empleos, utilizando los medios y recursos del territorio y Montoya (1998) considera que se basa en la identificación y aprovechamiento de los recursos y potencialidades endógenas de una comunidad, barrio o ciudad, se identifica que el sector educativo superior tecnológico puede contribuir al desarrollo local de las microempresas a través de la vinculación de sus sistemas educativos. Desde el punto de vista de la política de desarrollo económico y social de la pequeña y mediana empresa en México, Anzola (2003) define a MiPyme como aquella que merece apoyo pleno, pero no lo encuentra ni en las instituciones privadas ni en las oficinas públicas por su pequeñez, lo cual representa una dificultad para que las instituciones o mecanismos establecidos puedan auxiliarla en los aspectos administrativos, financieros, técnicos y de mercado. Desde el punto financiero se dice que es aquella que no es sujeta de crédito.

La Constitución de los Estados Unidos Mexicanos también contempla el desarrollo de las empresas en su artículo 25 al citar en sus párrafos:

La ley establecerá los mecanismos que faciliten la organización y la expansión de la actividad económica del sector social, abarcando todos los sectores productivos 0 no y, en general, de todas las formas de organización social para la producción, distribución y consumo de bienes y servicios, y protege la actividad económica que realicen los particulares y provee condiciones para que el desenvolvimiento del sector privado contribuya al desarrollo económico nacional, im-
Anzola (2003) define a MiPyme como aquella que merece apoyo pleno, pero no lo encuentra ni en las instituciones privadas ni en las oficinas públicas por su pequeñez, desde el punto financiero no es sujeta de crédito 
plementando una política nacional para el desarrollo industrial sustentable que incluya las vertientes sectoriales y regionales.

\section{SELECCIÓN DE LA MUESTRA}

En este trabajo no se utilizó una muestra, ya que el criterio de selección de las microempresas fue la aplicación de los PI por parte de los estudiantes del Instituto Tecnológico Superior de Poza Rica en las microempresas locales, y debido a esta limitación la muestra poblacional no presenta los esquemas correspondientes, por tal razón el método seguido es No Probabilístico Intencional teniendo en cuenta los siguientes criterios:

- Los PI se desarrollaron con la asesoría de 3 asignaturas.

- Los estudiantes cursaban la carrera de Gestión Empresarial.

- Se consideró a estudiantes del Octavo Semestre (Febrero - Junio 2018).

- Se consideró a estudiantes del Séptimo Semestre (Agosto-Enero 2018).

- Se consideró a estudiantes del Semestre Febrero-Junio 2019.

- Se organizaron equipos de estudiantes y se realizaron los $\mathrm{PI}$ en 25 microempresas.

\section{INSTRUMENTO DE INVESTIGACIÓN}

Se aplicaron matrices MEFI, MEFE, FODA, encuestas de mercado, entrevistas a los representantes de las microempresas, diagramas de flujo, se realizaron encuestas a los empresarios y representantes de las microempresas.

\section{PROCEDIMIENTO}

Se empleó el proceso de proyectos integradores para la formación y desarrollo de competencias profesionales del Tecnológico Nacional de México (2014) y consiste en una estrategia metodológica y evaluativa de investigación, direccionada al planteamiento y solución de problemas y requiere de la articulación de asignaturas del nivel y disciplina o carrera, considerando:

1. Definición de la Asignatura Eje.

2. Selección de las competencias de cada asignatura que se vinculan al proyecto.

3. Definición del Nodo Problematizador.

De acuerdo al problema y las competencias de cada asignatura, se planearon técnicas de ingeniería y se aplicaron a las microempresas. Se analizaron y se entregó un informe técnico a cada una de las empresas. Se llevó a cabo la presentación ante la Academia de Ingeniería en Gestión y se realizó la evaluación mediante rúbricas.

Se realizó una encuesta a los representantes de las empresas, para conocer el impacto que generó la realización de los $\mathrm{Pl}$, posteriormente se analizaron las respuestas mediante la representación gráfica.

\section{RESULTADOS}

De las 25 microempresas en las que se realizó el PI, el $90 \%$ consideró que tuvo un impacto innovador, ya que no se les había aplicado nunca técnicas de ingeniería para detectar el nodo problematizador 0 áreas de mejora, y con ello asignar recursos en aquellas áreas que lo requieren; el $87 \%$ consideró que se le presentaron estrategias que podían cumplir a corto y mediano plazo; el 20\% de las microempresas habían implementado una estrategia tecnológica sin que al momento pudieran medir el impacto económico por el tiempo transcurrido en la im- plementación; el 92\% consideró que al contar con el PI tenía más claro la toma de decisiones para la mejora de su microempresa.

\section{CONCLUSIONES}

La participación de los estudiantes en las empresas mediante la realización de los PI permite establecer las siguientes conclusiones:

- Las empresas detectan sus áreas de mejora, lo que les permite planificar sus actividades para alcanzar los objetivos planteados a partir del diagnóstico realizado.

- Se optimiza la asignación de recursos.

- A mediano y largo plazo se incrementan las ventas y por ende, las utilidades.

- Se crea un vínculo que favorece al desarrollo local, a partir del crecimiento de las microempresas.

- Las empresas pueden desarrollan su capacidad de innovar, facilitar el proceso de la toma de decisiones, identificar áreas de mejora y utilizar los medios tecnológicos.

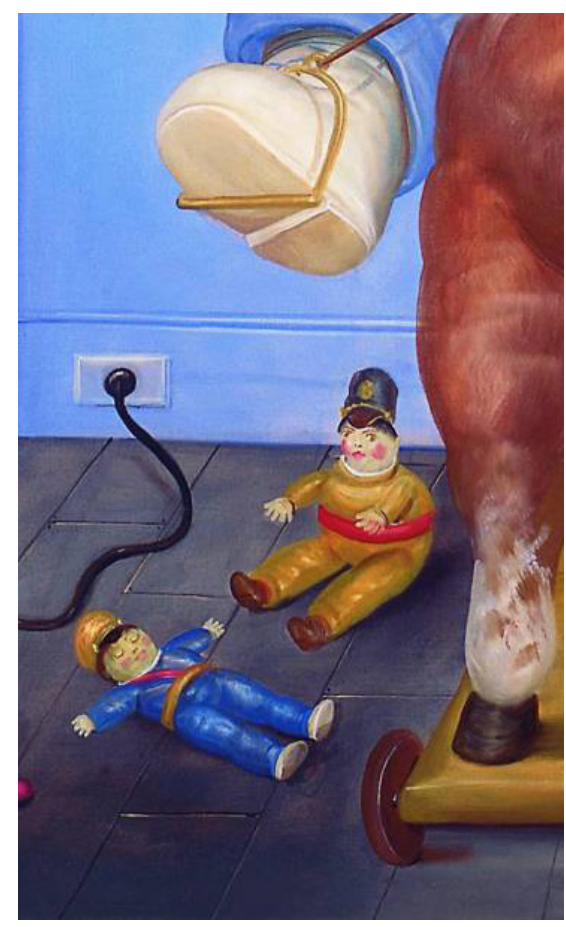




\section{REFERENCIAS}

Alburquerque, F. (2002). Desarrollo económico territorial. Guía para agentes. Instituto de Desarrollo Regional, Fundación Universitaria, Sevilla.

Anzola, R. S., (2003). Administración de pequeñas empresas. CDMX, México: Mc Graw Hill.

Dirección General de Educación Superior Tecnológica (2012). Modelo Educativo para el Siglo XXI: Formación y desarrollo de competencias profesionales. México, D.F.: Sfera Creativa, S.A. de C.V.

López Rodríguez, N. M. (2012). El proyecto Integrador: Estrategia didáctica para la formación de competencias desde la perspectiva del enfoque socioformativo. México: Gafra Editores.

Lineamientos Académico-Administrativos versión 1.0, para los planes y programas de estudio 2009-2010. México.

Molina Ruíz, E. (2007). La Práctica Profesional, componente de formación en la preparación de futuros profesionales. Investigación Educativa, 11(19), 19-34. Obtenido de http://go.galegroup.com.ezproxylocal.library.nova.edu/ps/retrieve

Rascón Chávez, 0. (2010). Prospectiva de la Ingeniería en México y en el Mundo. Academia de Ingeniería México. México, D.F.

Saldaña R. J. (2005). Recomendaciones para la implantación de la herramienta Balances Scorecard en las PYMES (Tesis de maestría). Tecnológico de Monterrey, Monterrey, México.

Tecnológico Nacional de México (2014). Proyectos Integradores para la formación y desarrollo de competencias profesionales ( $2^{\mathrm{a}}$. edición.). México.

Tobón, S.P. (2010a). Secuencias didácticas: aprendizaje y evaluación de competencias. México: Pearson.

Tobón, S.y. (2010b). El modelo de competencias en las prácticas docentes: Hacia escenarios significativos de vida. México: Conrrumbo.

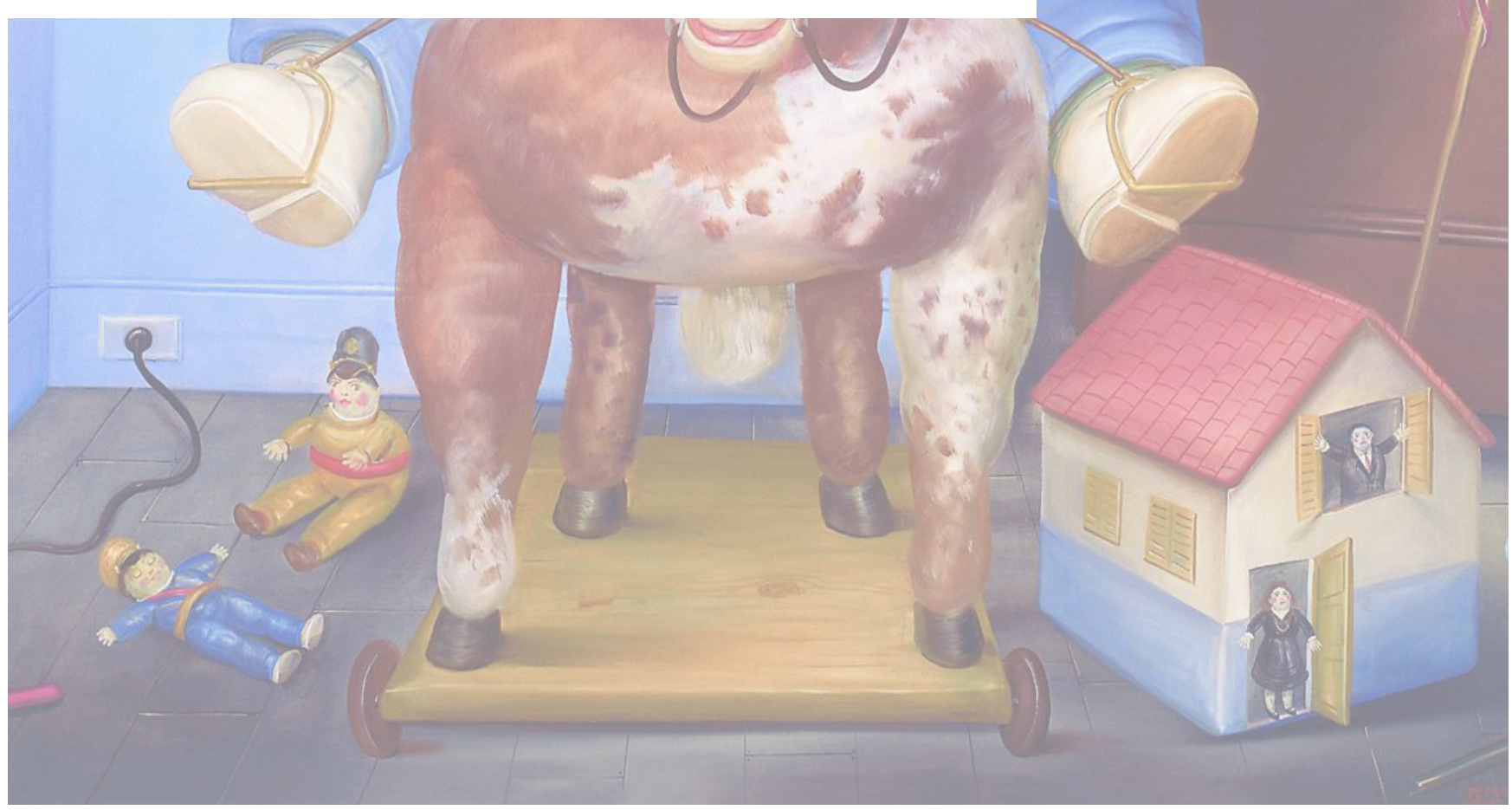




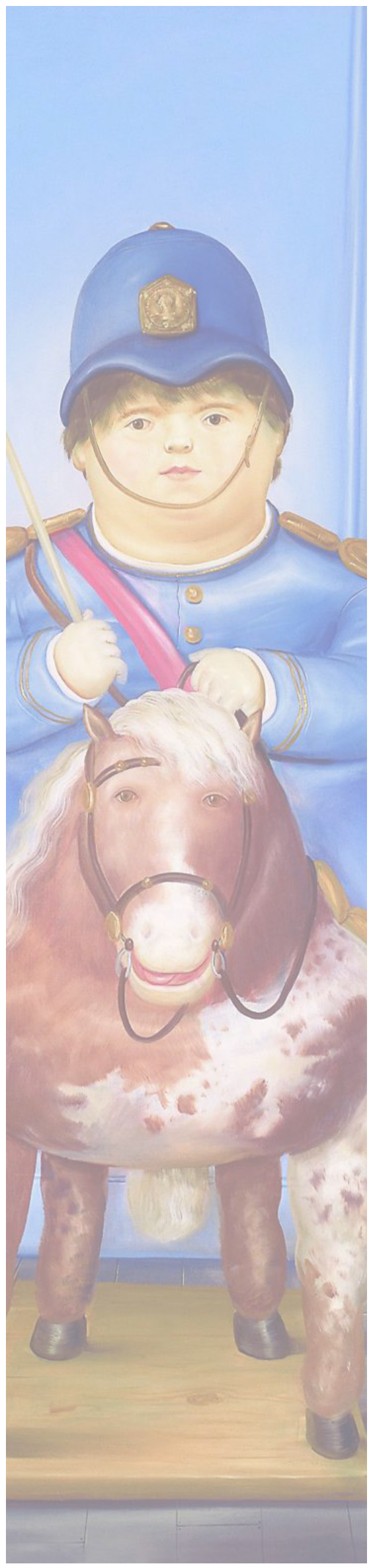

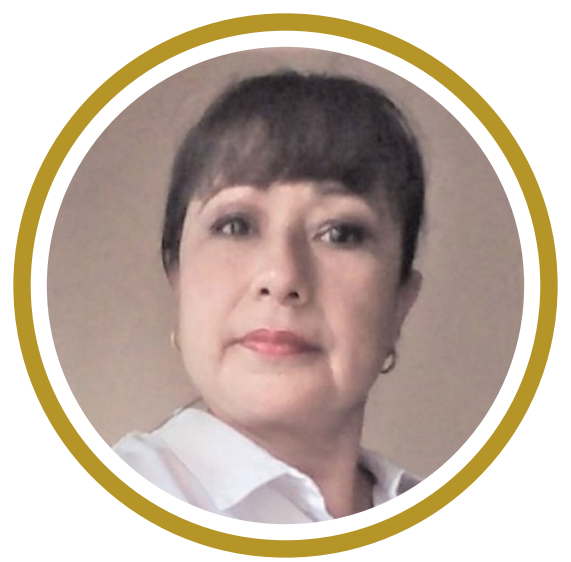

\section{Maribel \\ Martínez Lee}

Licenciada en Derecho y en la Especialidad en Ciencias Sociales, cuenta con una maestría en Ciencias de la Administración por parte de la Universidad del Valle de México. Trabaja como Profesora de la carrera de Ingeniería en Gestion Empresarial del Tecnológico Nacional de México, unidad Poza Rica del Estado de Veracruz. Tiene participaciones en trabajos de investigación en el área educativo y empresarial.

\section{Correo Electrónico:}

maribel.martinez@itspozarica.edu.mx

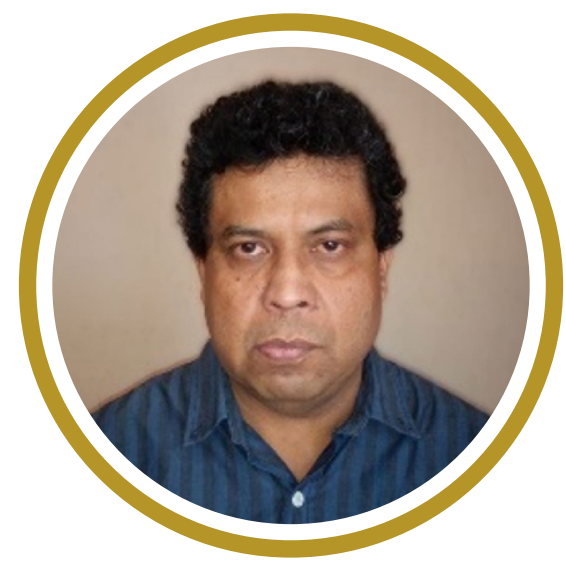

Celso Ramón Sarmiento Reyes

Licenciado en Economía, Maestro en Economía Aplicada y Doctor en Ciencias Sociales. Ha colaborado en diversas instituciones educativas como profesor-investigador. Sus líneas de investigación son el Desarrollo Regional, Turismo, Empresas, y Políticas Públicas. Ha publicado artículos sobre Desarrollo Regional, desde diversas perspectivas. Actualmente es Profesor a Tiempo Completo del Instituto Tecnológico Superior de Poza Rica.

\section{Correo Electrónico:}

celso.sarmiento@itspozarica.edu.mx

Fecha de Recepción: 15/03/20

Fecha de Aprobación: 15/08/20 